\title{
Impact of modern personalized treatment of breast cancer on surgical attitude and outcomes
}

\author{
CIPRIAN TĂNĂSESCU ${ }^{1,2}$, DRAGOS SERBAN ${ }^{3,4}$, ANDREI MOISIN ${ }^{2}$, \\ CARMEN POPA $^{2}$, RAMONA COCA ${ }^{1,2}$, GEORGE IANCU ${ }^{5,6}$, MIHAIL SILVIU TUDOSIE $^{7,8}$, \\ DANIEL OVIDIU COSTEA ${ }^{9,10}$, BOGDAN SOCEA ${ }^{3,11}$, CORNELIU TUDOR $^{4}$, GABRIEL ANDREI GANGURA $^{3,12}$, \\ LAURA CARINA TRIBUS ${ }^{13,14}$ and GABRIEL CATALIN SMARANDACHE ${ }^{3,4}$
}

${ }^{1}$ Surgical Clinical Department, Faculty of Medicine, 'Lucian Blaga' University, 550169 Sibiu; ${ }^{2}$ Department of Surgery, Sibiu County Clinical Emergency Hospital, 550245 Sibiu; ${ }^{3}$ Department of General Surgery, Faculty of Medicine,

'Carol Davila' University of Medicine and Pharmacy, 020021 Bucharest; ${ }^{4} \mathrm{IV}$ th Department of Surgery, Emergency University Hospital, 050098 Bucharest; ${ }^{5}$ Department of Obstetrics and Gynecology, 'Filantropia' Clinical Hospital, 011132 Bucharest; ${ }^{6}$ Department of Obstetrics and Gynecology, Faculty Of Medicine, and ${ }^{7}$ Department of Clinical Toxicology, Faculty Of Medicine, 'Carol Davila' University of Medicine and Pharmacy, 020021 Bucharest; ${ }^{8}$ ICU II Toxicology, Clinical Emergency Hospital, 014461 Bucharest; ${ }^{9}$ Department of Clinical Surgical Disciplines I, Faculty of Medicine, 'Ovidius' University, 900470 Constanta; ${ }^{10}$ First Surgery Department, Emergency County Hospital, 900591 Constanta; ${ }^{11}$ Department of Surgery, 'Sf. Pantelimon' Emergency Hospital, 021659 Bucharest;

${ }^{12}$ Second Department of Surgery, Emergency University Hospital, 050098 Bucharest; ${ }^{13}$ Department of Internal Medicine Gastroenterology, Faculty of Medicine, 'Carol Davila' University of Medicine and Pharmacy, 020021 Bucharest; ${ }^{14}$ Department of Gastroenterology, Emergency University Hospital, 050098 Bucharest, Romania

Received August 30, 2021; Accepted September 29, 2021

DOI: $10.3892 / \mathrm{etm} .2021 .10979$

\begin{abstract}
Multimodal treatment of breast cancer has made steady progress in recent years. The involvement of modern oncology, diagnostic imaging techniques and surgical treatment, have brought a definite benefit to patients, defining the multidisciplinary treatment of breast cancer. The introduction of immunohistochemical testing and genetic screening has led to the prioritization of therapy according to their results and a correct approach to initiating treatment. The main aim of the present study was to conduct a comparative analysis through a retrospective study of the therapeutic means used in breast cancer with the statistical evaluation of the obtained results. To carry out the study, a group of 125 patients hospitalized during the period January 2015 to December 2020, were included, and the parameters were selected from the observation sheets. The results of the study demonstrated the superiority of multimodal treatment of breast cancer over surgical treatment as the only therapeutic management. The introduction of ultrasound-guided biopsies and conservative surgical options
\end{abstract}

Correspondence to: Dr Dragos Serban, Department of General Surgery, Faculty of Medicine, 'Carol Davila' University of Medicine and Pharmacy, 37 Dionisie Lupu Street, 020021 Bucharest, Romania E-mail: dragos.serban@umfcd.ro

Key words: breast cancer, multimodal therapy, surgery, neoadjuvant chemotherapy, immunohistochemical analysis, outcomes has led to increased diagnostic accuracy and a significant improvement in aesthetic outcome. The multidisciplinary approach to breast cancer allows an individualized treatment by performing immunohistochemical testing and through the use of neoadjuvant and adjuvant treatment combined with conservative surgical techniques with a more favorable cosmetic and oncological result, with reduced postoperative complications.

\section{Introduction}

In Romania, the incidence of breast cancer is constantly on the increase with a higher distribution in urban areas, being the first cause of mortality (8.68\%) in women aged between 15 and 49 years and the third cause for women aged 50-69 years, after cardiovascular pathology (1).

Treatment in breast cancer is multidisciplinary, being individualized according to several factors such as the histological type of the tumor, its size, the presence or absence of lymphadenopathy, the patient's age and other associated risk factors or comorbidities. The treatment of choice remains surgery in combination with adjuvant therapies such as hormone therapy, immunotherapy, radiotherapy or chemotherapy, considerably increasing life expectancy, especially in the early stages of the disease. In the late stages, the primary aim is palliation and improving the quality of life. Early diagnosis is vital, thus periodic mammographic evaluation of the female population is the main way in which early forms of breast cancer can be detected. Confirmation of the diagnosis of malignancy is 
the first and most important stage in the management of this disease, being decisive for the patient's prognosis (2).

Surgical resection is one of the first effective treatments utilized in breast cancer and continues to play a critical role in the treatment of this pathology. The current standard is the multidisciplinary approach involving a coordinated effort of the surgeon and oncologist to achieve the best possible result.

The main aim of the present study was a comparative analysis of patients who have benefited from modern multimodal treatment versus those who have benefited exclusively from surgical treatment. At the same time, the importance of the applicability of hormonal treatment depending on the immunohistochemical result and the adjuvant radiotherapy treatment was considered, in order to reduce the loco-regional recurrence.

Given the continuous advances in modern breast biopsy techniques, a comparative analysis of the patients included in the study group was performed depending on the type of biopsy performed. The comparative analysis was also followed up depending on the type of surgery and the impact on postoperative complications.

\section{Patients and methods}

Patient data. The present study is an extensive retrospective one on 125 patients with breast cancer hospitalized between January 2015 and December 2020, in the First Surgery Department, Sibiu County Emergency Clinical Hospital (Sibiu, Romania). The statistical research was performed based on data from the analysis of observation sheets from the SCJU Sibiu archive, operating protocols, laboratory and intensive care. Patients diagnosed with intraoperative and preoperative breast cancer based on biological and imaging investigations were included in the study. Breast cancer patients who underwent prior surgery and presented to our clinic directly with relapses, cases in which patients refused surgical treatment, as well as patients diagnosed with benign tumors, were excluded.

A study protocol was developed to analyze the particularities of each patient diagnosed with breast cancer, selecting the necessary data from the observation sheet. Several parameters, including the annual distribution of breast cancer cases, patient demographics (age and environment), topographic location of the tumor, unilateral or bilateral involvement, staging, adjuvant/neoadjuvant chemotherapy, type of biopsy, type of surgery, associated comorbidities, type of oncological treatment applied depending on the immunohistochemical result, including chemotherapy, biologic therapy and hormone therapy, histological type of tumor and immediate postoperative complications, were evaluated. Depending on these parameters, the evolution and postoperative prognosis of the patients in the study group were analyzed.

Ethics approval and consent. The study was conducted according to the World Medical Association Declaration of Helsinki, and was waived for ethical approval due to its retrospective nature. Written informed consent was obtained from all 125 patients during the hospitalization.

\section{Results}

Demographic and clinical evaluation. A total of 125 patients that underwent surgery for breast cancer between January 2015 and December 2020, were included in the study group. There was a constant increase in the number of breast cancer cases admitted to our clinic during the study period from 13 cases admitted in 2015 (10.4\%) to 42 cases in 2020 (33.6\%). One explanation may be the employment of the national screening program, which allowed early diagnosis. On the other hand, epidemiological studies showed a global increase in the incidence of breast cancer in recent years, due both to higher addressability and the development of screening modalities over time (3).

Regarding the demographic data of the patients, a higher number of breast cancer patients were from urban areas, i.e., 64 cases $(77 \%)$ compared to rural areas, i.e., 19 cases $(23 \%)$. This was explained by the more developed health education system in the urban areas, the greater accessibility of patients to medical services and the possibility of earlier diagnosis.

Of the 125 patients included in the study group, the majority were aged $51-70$ years $(52.8 \%)$, with a mean age of 67.3 years (Table I). The most frequent location was encountered in the supero-external quadrant $(48 \%)$, followed by the infero-external quadrant (20.8\%). This distribution coincides with previously published findings, the most common location of tumor formation being in the supero-external quadrant and being associated with an improved prognosis, while tumors in the central quadrant were associated with a more reserved prognosis and mortality that was twice as high, most likely due to later diagnosis of neoplasms with this localization (4). Bilateral involvement was diagnosed in $21 \%$, more often in advanced cases and involving a genetic component (Table I).

Staging of breast cancer is a mandatory step in the diagnosis of this pathology, as it is essential to establish therapeutic behavior and prognosis of patients. For the analysis of the distribution of cases according to the stage of the disease, the p-TNM staging indicated by the histopathological examination was used. In the present study, most cases were diagnosed in stage III disease (35.2\%) and only $9.6 \%$ of cases were diagnosed in the early stages of carcinoma in situ. The early stages at the time of diagnosis have the advantage of a more favorable prognosis and benefit from much less invasive treatments. The diagnosis of breast cancer in advanced stages can be explained by the lack of national screening programs, low level of education on maintaining health and the importance of self-examination, and difficult access to medical services and treatments. Thus, late diagnosis has major consequences on the survival rate and quality of life, correlating with more invasive treatments $(5,6)$.

Breast cancer biopsy is the gold standard for pathological evaluation guiding the therapeutic conduct for each case. In the present study group, of the 125 cases, 55 (44\%) received a biopsy at our department, while for the remaining patients the biopsy was performed in other departments, prior to admission. The types of biopsies performed included incisional, excisional biopsy, most patients benefited from incisional biopsy (36.4\%), followed by excisional biopsy (23.6\%). Ultrasound guided core needle biopsy $(21.8 \%)$ and fine needle aspiration biopsy (18.2\%) were also used (Table I). This result 
Table I. Demographic and clinical evaluation of the patients in the study group.

\begin{tabular}{|c|c|}
\hline Parameter & No $(\%)$ \\
\hline \multicolumn{2}{|l|}{ Age } \\
\hline$<40$ & $8(6.4)$ \\
\hline $41-50$ & $20(16)$ \\
\hline $51-60$ & $27(21.6)$ \\
\hline $61-70$ & $39(31.2)$ \\
\hline $71-80$ & $22(17.6)$ \\
\hline$>80$ & $9(7.2)$ \\
\hline \multicolumn{2}{|l|}{ Comorbidities } \\
\hline Heart disease & $68(54.4)$ \\
\hline Liver disease & $3(2.4)$ \\
\hline Neurological disorder & $16(12.8)$ \\
\hline Diabetes & $21(16.8)$ \\
\hline Hypothyroidism & $2(1.6)$ \\
\hline Chronic renal failure & $3(2.4)$ \\
\hline Obesity & $12(9.6)$ \\
\hline \multicolumn{2}{|l|}{ Type of biopsy } \\
\hline Incisional biopsy & $45(36.4)$ \\
\hline Excisional biopsy & $30(23.6)$ \\
\hline Ultrasound guided core needle biopsy & $27(21.8)$ \\
\hline Fine needle aspiration biopsy & $23(18.2)$ \\
\hline \multicolumn{2}{|l|}{ Tumor location (quadrants) } \\
\hline Supero-external & $60(48)$ \\
\hline Infero-external & $26(20.8)$ \\
\hline Central & $19(15.2)$ \\
\hline Supero-internal & $11(8.8)$ \\
\hline Infero-internal & $9(7.2)$ \\
\hline \multicolumn{2}{|l|}{ Uni-/bilateral involvement } \\
\hline Unilateral & $99(79)$ \\
\hline Bilateral & $26(21)$ \\
\hline \multicolumn{2}{|l|}{ Histopathological type } \\
\hline Invasive ductal carcinoma (NST) & $94(75)$ \\
\hline Invasive lobular carcinoma & $19(15)$ \\
\hline Mixed or unassociated carcinomas & $12(10)$ \\
\hline \multicolumn{2}{|l|}{ Staging (p-TNM) } \\
\hline Tis & $12(9.6)$ \\
\hline I & $16(12.8)$ \\
\hline II & $29(23.2)$ \\
\hline III & $44(35.2)$ \\
\hline IV & $24(19.2)$ \\
\hline Neoadjuvant therapy & $101 / 125(81)$ \\
\hline \multicolumn{2}{|l|}{ Chemotherapy } \\
\hline Early stages & $48 / 57(84.2)$ \\
\hline Advanced stages & $53 / 68(77.9)$ \\
\hline Chemotherapy + biologic therapy anti Her-2 & $21(16.8)$ \\
\hline Chemotherapy + hormone therapy & $38(30.4)$ \\
\hline
\end{tabular}

coincided with data from the literature, incisional biopsy being indicated if the lesion was highly likely to be malignant or could not be reached through needle biopsy. However, while surgical methods of biopsy have a higher degree of accuracy, they also have a higher degree of complications, such as bleeding or infection compared to needle biopsy $(7,8)$.

Recent findings, however, have shown that imaging-guided percutaneous breast biopsies have almost completely replaced diagnostic surgical excisions that have been associated with longer hospitalization, higher costs, and possible complications (9). In 2010, the European Society of Breast Cancer Specialists, EUSOMA, suggested that $90 \%$ of women with breast cancer (invasive or ductal carcinoma in situ) should have a preoperative diagnosis by percutaneous biopsy, which is essential in the management of breast lesions (9).

Histopathological examination plays an important role in selecting the therapeutic strategy in patients with breast cancer that allows detailed analysis of the samples sent (biopsy/surgery). The correct approach of the samples requires the integration of both clinical and imaging results. The result obtained after the histopathological examination, guides the therapeutic options for each patient. The appearance of modern molecular techniques has suggested the disappearance of histopathological examinations. However, currently and in the near future, this examination remains a key element of the integrated multidisciplinary team involved in the treatment of patients with malignant breast disease (10). Considering the importance of the histopathological analysis, the analysis of the study group according to this result was deemed crucial, with the highlighting of the histological types of breast neoplasm (Table I). Thus, of the total number of patients included in the group, most were diagnosed with NST invasive carcinoma representing $75 \%$ of the total group, followed by invasive ductal carcinoma identified in 19 patients, representing $15 \%$ of the total group. Other types of neoplasms, mixed or non-associated, were identified in 12 patients. These results coincided with data from the literature mentioning invasive ductal carcinoma NST as the most common form of invasive breast cancer (11).

The treatment of breast cancer has evolved over time from a strictly surgical approach with an emphasis on loco-regional disease control to multidisciplinary management, with an emphasis on systemic therapy, significantly improving survival. The optimal time of radio-chemotherapy in relation to operating time has been studied for several decades, with solid evidence supporting the neoadjuvant approach (12). The study group was divided into two groups to evaluate the applicability of neoadjuvant chemotherapy. Of the total patients included in the group, $101(81 \%)$ benefited from neoadjuvant chemotherapy, of which $84.2 \%$ of the patients were diagnosed in early stages and $77.9 \%$ of the patients were diagnosed in advanced stages (Table I). Notably, in the early stages, the percentage of patients who did not benefit from neo-adjuvant chemotherapy was lower than that of patients in advanced stages; 21 patients received biologic treatment with anti-HER-2 in combination with neoadjuvant chemotherapy. Neoadjuvant hormone therapy for intensely positive hormone receptors but with HER-2 negative, was applied to 38 patients. Most patients received sequential neoadjuvant chemotherapy, of whom 15 for early breast cancer and 27 for loco-regional advanced cancer (Table I).

Surgical treatment in breast cancer plays a central role, aiming at both the resection of the tumor in oncological limits 
Table II. Annual distribution of performed surgeries and complications in the study group.

\begin{tabular}{lccccccc}
\hline & \multicolumn{7}{c}{ Year } \\
\cline { 2 - 7 } Performed surgeries/complications & $2015(\%)$ & $2016(\%)$ & $2017(\%)$ & $2018(\%)$ & $2019(\%)$ & $2020(\%)$ & Total \\
\hline No. of surgeries for breast cancer, n (\%) & $13(10.4)$ & $14(11.2)$ & $15(12)$ & $15(12)$ & $26(20.8)$ & $42(33.6)$ & 125 \\
No. of conservative surgeries, n (\%) & $3(23)$ & $4(28.5)$ & $5(30)$ & $6(40)$ & $7(26.9)$ & $10(23.8)$ & 35 \\
Complications, n (\%) & $6(46)$ & $4(28.5)$ & $4(26.6)$ & $3(20)$ & $1(3.8)$ & $1(2.3)$ & $19(15.2 \%)$ \\
\hline
\end{tabular}

and a favorably aesthetic appearance. In the studied group, the choice of surgical therapy was made depending on the stage, histological type and size of the tumor, the general condition, associated comorbidities and the patient's decision. Of note is the fact that in the therapeutic decision of breast cancer, the solution offered by the oncology commission had a categorical role, each patient being presented to the oncology commission in order to choose the optimal individualized therapeutic option. The most used surgical technique was the Madden modified radical mastectomy, performed in $54.16 \%$ of patients, which was associated with a lower rate of postoperative complications and is less mutilating, preserving both pectoral muscles compared to other radical surgical techniques (13).

In 35 cases, conservative surgery was performed, respectively, sectorectomy followed by axillary lymphadenectomy (28\%), followed by a cleaning mastectomy performed in 10 patients and a complementary axillary lymphadenectomy, which was performed in 6 cases due to local recurrence. The lowest percentage was recorded at the modified radical Patey mastectomy, i.e., 3 patients (2.4\%) (Fig. 1).

The constant, slow growth each year included in the current study, of conservative surgeries to the detriment of the radical classical techniques is crucial. In 2015, 3 conservative surgeries were performed, but they increased steadily to a peak in 2020 when 10 conservative surgeries were performed (Table II). The results obtained were consistent with other clinical studies that showed a steady increase in the number of patients receiving conservative surgery over conventional ones (14).

Associated comorbidities with accompanying life risk play an important role in the prognosis and postoperative evolution of the disease. These data correspond to those in the specialized articles, the high rate of associated cardiovascular diseases can be explained by the advanced age at which the neoplastic pathology was diagnosed. There was also a significant increase in mortality and an improved prognosis in patients with cardiovascular disease and diabetes. The presence of these pathologies requires a multidisciplinary approach and a much more complex therapeutic strategy (15).

The most common immediate postoperative complications in breast cancer are local. In the analyzed group, immediate postoperative complications were present in 19 patients, representing $15.2 \%$ of the studied group. Among the complications, the most common was prolonged lymphorrhagia, present in 13 patients $(68.4 \%)$, while in 3 patients $(15.8 \%)$ there was postoperative hematoma. The rarest complications were represented by the wound infection: 2 cases $(10.5 \%)$ and the axillary lymphocele: 1 case $(5.3 \%)$. The results obtained were

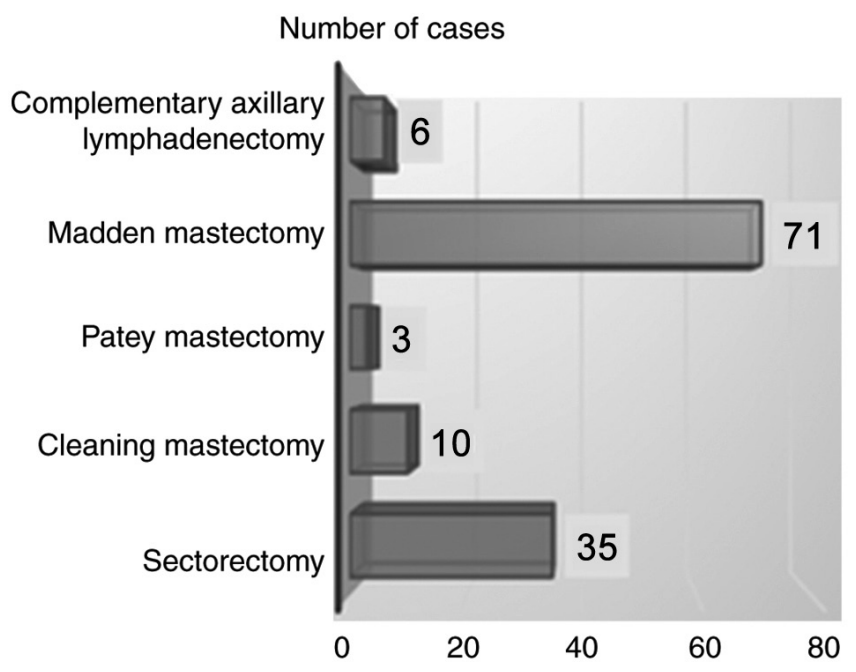

Figure 1. Distribution of patients according to the type of surgery.

partially correlated with recent data from the literature indicating seroma as the most common postoperative complication followed by wound infection, hematoma and flap necrosis (16). Of note is the absence of seroma in patients who underwent surgery, which can be explained by the use of drainage tubes at the end of the surgery.

The study group was analyzed according to the annual incidence of immediate postoperative complications. Thus, the majority of complications occurred during the period 2015-2017 (14 patients) and the minority, in 2019 and 2020, each with one case (Table II).

Correlating these results with the type of modern surgery, an annual decrease in the number of immediate and late postoperative complications can be observed, with the annual increase in the number of conservative interventions. These results coincide with specialized studies showing a decrease in the number of patients with postoperative complications with the introduction of conservative surgery (17). Following a comparative study of postoperative complications after mastectomy and conservative surgery, the latter has been shown to be superior to modified mastectomy in the incidence of postoperative complications (18). Radiation therapy (RT) is an integrated component of the multidisciplinary management of breast cancer, significantly reducing locoregional recurrence and improving overall survival in both patients undergoing breast-conserving surgery (BCS) and patients with advanced breast cancer in which mastectomy is performed (19). All 125 patients in the study group underwent radiotherapy, of whom 
$80 \%$ received adjuvant radiotherapy and 25 patients $(20 \%)$ received palliative radiotherapy.

These results correlate with specialized studies demonstrating the efficacy of adjuvant radiotherapy by improving local disease control and overall survival after mastectomy (20). In addition, some randomized studies have shown that adjuvant radiotherapy after conservative breast surgery results in overall long-term survival, which are comparable to modified radical mastectomy. Postoperative radiotherapy leads to a significant reduction in local recurrences compared to conservative surgical treatment, used as the only therapeutic option. A recent meta-analysis of the 'Early Breast Cancer Trialists Collaborative Group (EBCTCG)' highlighted the major advantage of adjuvant radiotherapy after conservative surgery in terms of survival in positive lymph node breast cancer. For most older women diagnosed with early breast cancer, the current standard treatment after BCS is adjuvant radiotherapy and adjuvant endocrine therapy (21).

\section{Discussion}

In the present study, most of the patients were aged between 50 and 70 years, which could be explained by longer exposure to risk factors for breast cancer. Although breast cancers diagnosed at older ages are more common and more numerous, they have an improved prognosis, progress more slowly, and are less aggressive than cancers diagnosed at a young age, where the genetic component plays a key role (22).

Detection of BRCA1 and BRCA2 mutations can lead to both a decrease in the incidence of breast cancer and a decrease in morbidity through early detection. In addition, the early age associated with the mutation of these genes has a major impact on contralateral breast cancers (23). Previous findings confirm the existence of an increased risk of contralateral breast cancer in patients with this disease from families with mutations in the BRCA1 and BRCA2 genes (23). However, little information is available on bilateral breast cancer in patients from families at high risk for this disease but with negative results for $B R C A 1$ and $B R C A 2$ gene mutations. A large multicenter retrospective study of a group of 6,235 women with unilateral breast cancer from high-risk families showed that the risk of contralateral breast cancer was significantly higher in patients from families with $B R C A I$ mutation compared to patients from families with the $B R C A 2$ gene mutation. In comparison, patients from families testing negative for the BRCA 1-2 gene mutation have a much lower risk of developing contralateral breast cancer (23). The same study suggests that the cumulative risk of contralateral breast cancer, 25 years after the first breast cancer, was $44.1 \%$ in $B R C A 1$-positive patients, $33.5 \%$ in BRCA2-positive patients, and only $17 \%$ in family patients. tested negative for the $B R C A$ genes. Family members with the BRCAl mutation had a 3 -fold higher risk of breast cancer contralateral than family members without the BRCA1-2 mutations (23).

Additionally, early age in the first breast cancer was associated with a higher risk of bilateralization. In patients who developed breast cancer prior to the age of 40 , the cumulative risk of contralateral breast cancer after 25 years was $>55 \%$ for $B R C A 1,>38 \%$ for $B R C A 2$, and only $28.4 \%$ for patients from families testing negative for the BRCAl and 2 genes.
Compared to patients $>50$ years of age, the cumulative risk of contralateral breast cancer after 25 years was $>21 \%$ for $B R C A 1,>15 \%$ for $B R C A 2$ and $12.9 \%$ in patients from $B R C A 1$ and 2-negative families (23).

The therapy of patients with breast cancer involves three main treatment modalities: surgical, systemic therapy and radiotherapy. According to the national protocols, systemic therapy was administered to patients with breast cancer after surgery. However, previous findings suggest that systemic neoadjuvant therapy (NA) is an equally effective option compared to adjuvant therapy. Although neoadjuvant anti-hormone therapy is recommended mainly for patients with positive hormone receptors in the postmenopausal period, neoadjuvant chemotherapy is increasingly used for all subtypes of breast cancer. It increases the rate of breast preservation surgery and allows monitoring of the response to treatment by providing unique opportunities for the development of both individualized treatment strategies and the development of new generation drugs. Modern treatment strategies are adapted to molecular subtypes, allowing a more individualized approach to therapy (24).

Tumor biopsy in the mammary gland is essential in the diagnosis of breast cancer. Although it is a more or less invasive method (depending on the type of biopsy), it allows the diagnosis of certainty. In addition, it provides information on the type of cancer and the degree of tumor differentiation. The biopsy complements the information obtained from the clinical and imaging examination so that the patient can benefit from a correct and targeted treatment depending on the outcome of the histopathological examination (7).

The cytological and histological diagnosis of a palpable/nonpalpable mass can be obtained by performing a percutaneous biopsy [fine needle aspiration biopsy (FNA) or cutting needle biopsy (CNB)] or surgically (incisional or excisional biopsy). Among the main objectives of percutaneous biopsy techniques are: achieving the maximum degree of accuracy and providing as much information about the tumor (histological type, degree of differentiation, invasion, immunohistochemical data). To achieve these goals, percutaneous biopsy devices have evolved from fine needle aspiration, to CNB and later vacuum-assisted biopsy (VAB) (8-10). Currently, ultrasound-guided breast biopsy has become the first choice for performing a percutaneous biopsy in most lesions detected by ultrasonography. The sensitivity of the ultrasound-guided biopsy is about $97.5 \%$, which makes this technique optimal for performing a breast biopsy. Ultrasound-guided needle biopsy (CNB) is a modern, safe and accurate technique that is currently considered the method of choice in all lesions classified as BI-RADS 3, 4 and 5, while stereotaxis and MRI should be reserved for lesions that are not clearly visible on ultrasound (25). Although incisional surgical biopsy was the most commonly used type of biopsy, the trend is to replace it with modern, minimally invasive techniques, represented by imaging-guided core-needle biopsies (CNBs) that are cost effective, and have fewer complications and high accuracy.

The histopathological examination of a breast biopsy or fragment of surgery must specify the histological type of the tumor, the histological grade and the performance of immunohistochemistry tests (26). The development and progression of breast cancer have been linked to the interaction between tumor 
cells and the microenvironment formed by breast cancer cells, fibroblasts, adipocytes, immune and endothelial cells (27). Angiogenesis is essential for the tumor to grow and it can be considered as a specific mark of the neoplastic process (28). Immunohistochemical testing is a special staining procedure performed on tissue fragments resulting from biopsy and which reveals the presence or absence of hormone receptors and/or HER2 receptors on the surface of cancer cells. This information plays an essential role in the subsequent planning of treatment (29). Hormone receptors (estrogenic-ER and progesterone-PR) are specialized proteins located on the surface or inside the neoplastic cell. Cells of certain tumors have a higher number of hormone receptors, thus indicating a favorable response to hormone therapy. Patients who are positive for these receptors have a more favorable prognosis and a higher survival rate (30). By contrast, patients with triple-negative breast cancer, who are negative for ER and PR and HER2 protein receptors have a more unfavorable prognosis and hormone therapy is ineffective in this case $(26,31)$. Women with only one type of positive receptor but with the other negative, can benefit from hormone therapy although the therapeutic response is variable $(32,33)$. The presence of tumor-infiltrating lymphocytes is another important factor of prognosis, being associated with a better neoadjuvant chemotherapy response and prognosis in HER2+ and HER2-breast cancers (34).

HER2 protein has a significant role in inducing breast carcinogenesis in vitro and in vivo. HER2 gene amplification or overexpression occurs in approximately $15-30 \%$ of cases of invasive breast cancer and has implications for both predictive and prognostic implications (10). Breast cancers with HER2/neu gene amplification or HER 2 protein overexpression are known as $H E R 2$-positive breast cancer and are associated with faster evolution and an increased risk of recurrence compared to Her2-negative neoplasm. HER2 should be determined in all patients with invasive breast cancer (9). Another useful test is the determination of the Ki67 marker. This index of cell proliferation is expressed as a percentage and is directly proportional to the proliferative activity of the neoplastic cell. Low levels are associated with low proliferative activity and high levels are associated with an unfavorable prognosis (11).

Genetic factors are known as the main etiopathogenic factors of breast cancer; thus, the genetic predisposition is responsible for 3-10\% of all cases of breast cancer and up to $30 \%$ of all those with early onset. Hereditary breast cancer is associated with mutations in the tumor suppressor genes BRCA1 (17q21) and BRCA2 (13q12-13) (34). This type of neoplasia has an autosomal dominant transmission, with more frequent appearance in an early form, of high intensity and with a tendency to become bilateral. Carriers of these mutations have a higher risk of developing this disease but also other types of neoplasms, such as ovarian cancer. These mutations are commonly seen in patients with a family history and those affected by multiple forms of the disease. Therefore, detecting mutations in these two genes is important in counselling family members and reducing the incidence of breast cancer. In the selection of patients for screening for germline mutations in the BRCAl gene, it is important to combine information about family history, age of diagnosis, and tumor morphology (34).
Hormonal factors are also correlated with the incidence of breast cancer. Yalagachin et al found that the prevalence of nodular goiter was higher in patients with malignant breast diseases than patients with benign breast diseases; however, the exact pathological mechanisms involved in these two diseases are still a subject of research (35).

A new risk factor for breast cancer was reported, i.e., breast augmentation surgery with silicone implant. In those cases, the histopathological aspect encountered was that of anaplastic large cell lymphoma (36). Surgeons should be aware of the association, as the number of plastic surgeries is currently an emerging trend.

A large part of the patients included in the study group received neoadjuvant systemic treatment. These are part of new treatment strategies adapted to molecular subtypes that allow an individualized approach in the administration of therapy.

Adjuvant radiotherapy reduces locoregional recurrence and improves overall survival, being an integral part of the multidisciplinary treatment of breast cancer.

Surgical resection was the first effective treatment for breast cancer and remains the most important treatment modality. Improvements in surgical techniques, along with the use of adjuvant radiotherapy and advanced chemotherapeutic agents, have helped to orient them towards more focused and, at the same time, less invasive surgical techniques. Surgical management of breast cancer has changed, from extensive procedures with a fairly high morbidity rate, to modern surgical techniques in order to achieve the most favorable possible cosmetic result in tandem with adequate cancer resection (37).

Recent clinical studies proved the importance of neoadjuvant therapy in improving the possibility of resectability and reducing intraoperative morbidity. In fact, neoadjuvant therapy has other roles, including that it increases the likelihood of conservative breast surgery in stage II-III patients who would otherwise require mastectomy due to an unfavorable ratio between breast and tumor formation, and reduces morbidity and the extent of lymph node in patients with significant axillary metastases. After neoadjuvant treatment, recommendations for surgery depend on the extent of the disease at presentation, the patient's decision, and the tumor response to systemic therapy. Modified radical mastectomy remains the standard for patients with inflammatory breast cancer, regardless of the response to neoadjuvant therapy $(12,38)$.

Conservative surgery for breast cancer aims to deliver a radical oncological result as effectively as possible, with negative resection margins confirmed histopathologically, but also with a favorable aesthetic appearance, which is extremely important for preventing psychological distress and body image disorders in breast cancer patients (39). Optimal local control of breast cancer by this method can be achieved only in combination with adjuvant radiotherapy, even if negative oncological margins have been obtained, because cancer cells may be present in the apparently unaffected breast tissue. In addition to being equivalent to radical mastectomy in terms of oncological safety, conservative surgery offers certain advantages in terms of quality of life and aesthetic results. This type of surgery allows both the preservation of the morphology of the breast and skin, as well as the preservation of sensitivity with a much lower psychological impact. Conservative surgery is currently the standard for patients with invasive breast 
cancer in stage 0 , I or II (40). However, some studies suggest a higher incidence of locoregional recurrence in patients treated with conservative surgery as opposed to those who received radical surgical treatment $(41,42)$.

Breast cancer continues to be a major public health problem, with its incidence continuing to rise both nationally and globally. Screening of patients with a genetic predisposition to breast cancer is important. Most of the patients included in the present study were diagnosed in advanced stages, with major consequences on survival rate and quality of life.

The treatment of breast cancer has undergone significant changes with the introduction of modern oncology and conservative surgical options. The involvement of a multidisciplinary team in this pathology represents the new therapeutic standard for both patients and clinicians, being individualized for each patient. The introduction of neoadjuvant chemotherapy in the treatment of breast cancer has led to an undeniable benefit both surgically and ontologically, it is becoming increasingly used for all subtypes of breast cancer, increasing the rate of conservative surgery. Immunohistochemical testing for the detection of HER 2 receptors, estrogenic and progesterone, has an essential role in the subsequent planning of individualized treatment.

Surgical treatment has been and will remain the most important treatment for curative purposes. The most used surgical technique was the modified Madden mastectomy, being preferred to other radical surgical techniques. There is a steady annual increase in the number of conservative surgeries to the detriment of the radical radicals, which highlights the current trend towards conservative surgery, being an integral part of modern treatment in breast cancer. Postoperative complications were present in a small number of patients, the most common being lymphorrhagia. The annual increase in the number of conservative surgeries is associated with an annual decrease in immediate and late postoperative complications.

\section{Acknowledgements}

Not applicable.

\section{Funding}

No funding was received.

\section{Availability of data and materials}

All data generated or analyzed during this study are included in this published article.

\section{Authors' contributions}

CiT, DS, AM, BS, DOC and CP were involved in the conception and design of this study. RC, AM, CP, GI, MST, CGS, LCT, CoT and GAG were responsible for the data collection and analysis. CiT, RC, CGS, LCT, MST and DS were responsible for drafting the manuscript. GI, DOC, BS, CoT and GAG provided critical perspective for important intellectual content. CiT, RC and AM are responsible for confirming the authenticity of the raw data. The final version was read and approved by all the authors.

\section{Ethics approval and consent to participate}

The study was conducted according to the World Medical Association Declaration of Helsinki, and was waived for ethical approval by the Bioethics Committee due to its retrospective nature. All patients previously signed written informed consent in regards to hospitalization and investigations.

\section{Patient consent for publication}

Not applicable.

\section{Competing interests}

The authors declare that they have no competing interests.

\section{References}

1. Furtunescu F, Bohiltea RE, Voinea S, Georgescu TA, Munteanu O, Neacsu A and Pop CS: Breast cancer mortality gaps in Romanian women compared to the EU after 10 years of accession: Is breast cancer screening a priority for action in Romania? (Review of the Statistics). Exp Ther Med 21: 268, 2021.

2. Brom L, Pasman HR, Widdershoven GA, van der Vorst MJ, Reijneveld JC, Postma TJ and Onwuteaka-Philipsen BD: Patients preferences for participation in treatment decision-making at the end of life: Qualitative interviews with advanced cancer patients. PLoS One 9: e100435, 2014.

3. Momenimovahed Z and Salehiniya H: Epidemiological characteristics of and risk factors for breast cancer in the world. Breast Cancer (Dove Med Press) 11: 151-164, 2019.

4. Rummel S, Hueman MT, Costantino N, Shriver CD and Ellsworth RE: Tumor location within the breast: Does tumour site have prognostic ability? Ecancermedicalscience 9: 552, 2015.

5. Caplan L: Delay in breast cancer: Implications for stage at diagnosis and survival. Front Public Health 2: 87, 2014.

6. Ben Fatma L, Belaid I, Said N, Gahbiche S, Hochlef M, Chabchoub I, Ezzairi F and Ben Ahmed S: What could be the reasons of late diagnosis of breast cancer in Tunisia? Tunis Med 96: 665-671, 2018.

7. Hegde V, Burke ZDC, Park HY,Zoller SD, Johansen D, Kelley BV, Levine B, Motamedi K, Federman NC, Seeger LL, et al: Is core needle biopsy reliable in differentiating between aggressive benign and malignant radiolucent bone tumors? Clin Orthop Relat Res 476: 568-577, 2018.

8. Zhang YJ, Wei L, Li J, Zheng YQ and Li XR: Status quo and development trend of breast biopsy technology. Gland Surg 2: 15-24, 2013.

9. Bick U, Trimboli RM, Athanasiou A, Balleyguier C, Baltzer PAT, Bernathova M, Borbély K, Brkljacic B, Carbonaro LA, Clauser P, et al: Image-guided breast biopsy and localisation: Recommendations for information to women and referring physicians by the European society of breast imaging. Insights Imaging 11: 12, 2020.

10. Hanby AM: The pathology of breast cancer and the role of the histopathology laboratory. Clin Oncol (R Coll Radiol) 17: 234-239, 2005.

11. Makki J: Diversity of breast carcinoma: Histological subtypes and clinical relevance. Clin Med Insights Pathol 8: 23-31, 2015.

12. Masood S: Neoadjuvant chemotherapy in breast cancers. Womens Health (Lond) 12: 480-491, 2016.

13. Feigenberg Z, Zer M and Dintsman M: Comparison of postoperative complications following radical and modified radical mastectomy. World J Surg 2: 207-211, 1977.

14. Morris CR, Cohen R, Schlag R and Wright WE: Increasing trends in the use of breast-conserving surgery in California. Am J Public Health 90: 281-284, 2000.

15. Nechuta S, Lu W, Zheng Y, Cai H, Bao PP, Gu K, Zheng W and Shu XO: Comorbidities and breast cancer survival: A report from the Shanghai breast cancer survival study. Breast Cancer Res Treat 139: 227-235, 2013.

16. Rizvi FH, Khan MK, Almas T, Ullah M, Shafi A, Murad MF, Ali A and Nadeem F: Early postoperative outcomes of breast cancer surgery in a developing country. Cureus 12: e9941, 2020. 
17. Hu C, Song H, Wang L, Jin L, Zhou X and Sun L: Clinical efficacy of anesthesia with intensive care nursing in attenuating postoperative complications in patients with breast cancer. J Int Med Res 48: 300060520930856, 2020.

18. Vishwakarma M and Sahani IS: Comparative study of complications of modified radical mastectomy and breast conservation therapy in early invasive breast cancer. Int J Surg Sci 3: 1-3, 2019

19. Jagsi R, Abrahamse P, Morrow M, Hawley ST, Griggs JJ, Graff JJ, Hamilton AS and Katz SJ: Patterns and correlates of adjuvant radiotherapy receipt after lumpectomy and after mastectomy for breast cancer. J Clin Oncol 28: 2396-2403, 2010.

20. Wöckel A, Wolters R, Wiegel T, Novopashenny I, Janni W, Kreienberg R, Wischnewsky M and Schwentner L; BRENDA study group: The impact of adjuvant radiotherapy on the survival of primary breast cancer patients: A retrospective multicenter cohort study of 8935 subjects. Ann Oncol 25: 628-632, 2014.

21. Matuschek C, Bölke E, Haussmann J, Mohrmann S, Nestle-Krämling C, Gerber PA, Corradini S, Orth K, Kammers K and Budach W: The benefit of adjuvant radiotherapy after breast conserving surgery in older patients with low risk breast cancer-a meta-analysis of randomized trias. Radiat Oncol 12: 60, 2017.

22. Han W, Kim SW, Park IA, Kang D, Kim SW, Youn YK, Oh SK Choe KJ and Noh DY: Young age: An independent risk factor for disease-free survival in women with operable breast cancer. BMC Cancer 4: 82, 2004

23. Rhiem K, Engel C, Graeser M, Zachariae S, Kast K, Kiechle M, Ditsch N, Janni W, Mundhenke C, Golatta M, et al: The risk of contralateral breast cancer in patients from BRCA1/2 negative high risk families as compared to patients from BRCA1 or BRCA2 positive families: A retrospective cohort study. Breast Cancer Res 14: R156, 2012

24. Untch M, Konecny GE, Paepke S and von Minckwitz G Current and future role of neoadjuvant therapy for breast cancer. Breast 23: 526-537, 2014.

25. Apesteguía L and Pina LJ: Ultrasound-guided core-needle biopsy of breast lesions. Insights Imaging 2: 493-500, 2011.

26. Perry N, Broeders M, de Wolf C, Törnberg S, Holland R and von Karsa L (eds): European guidelines for quality assurance in breast cancer screening and diagnosis. 4th edition. European Commission, Office for official publications of the European communities, Luxembourg, 2006. https://screening.iarc. fr/doc/ND7306954ENC_002.pdf. Accessed July 1, 2020.

27. Pantelimon I, Gales LN, Z̄gura A, Serbanescu GL, Georgescu DE, Nita I, Manolescu LSC, Stancu AM, Gruia MI, Anghel RM and Badiu DC: Analysis of oxidative stress in patients with breast cancer and obesity. Ann Med Health Sci Res 11: 1578-1585, 2021.

28. Ardeleanu V, Georgescu C, Frincu LD, Frincu LL and Vesa C: Angiogenesis as prospective molecular biology technique for cancer study. Rom Biotech Lett 19: 9637-9648, 2014.

29. Williams SL, Birdsong GG, Cohen $\mathrm{C}$ and Siddiqui MT: Immunohistochemical detection of estrogen and progesterone receptor and HER2 expression in breast carcinomas: Comparison of cell block and tissue block preparations. Int J Clin Exp Pathol 2: 476-480, 2009.
30. Sleightholm R, Neilsen BK, Elkhatib S, Flores L, Dukkipati S, Zhao R, Choudhury S, Gardner B, Carmichael J, Smith L, et al: Percentage of hormone receptor positivity in breast cancer provides prognostic value: A single-institute study. J Clin Med Res 13: 9-19, 2021.

31. Bacinschi XE, Zgura A, Safta I and Anghel R: Biomolecular factors represented by Bcl-2, p53, and tumor-infiltrating lymphocytes predict response for adjuvant anthracycline chemotherapy in patients with early triple-negative breast cancer. Cancer Manag Res 12: 11965-11971, 2020.

32. El Sayed R, El Jamal L, El Iskandarani S, Kort J, Abdel Salam M and Assi H: Endocrine and targeted therapy for hormone-receptor-positive, HER2-negative advanced breast cancer: Insights to sequencing treatment and overcoming resistance based on clinical trials. Front Oncol 9: 510, 2019.

33. Mehrgou A and Akouchekian M: The importance of BRCA1 and BRCA2 genes mutations in breast cancer development. Med J Islam Repub Iran 30: 369, 2016.

34. Mehedintu C, Bratila E, Berceanu C, Cirstoiu MM, Barac RI, Andreescu CV, Badiu DC, Gales L, Zgura A and Bumbu AG: Comparison of tumor-infiltrating lymphocytes between primary and metastatic tumors in Her2+ and HER2-breast cancer patients. Rev Chim 69: 3133-3137, 2018.

35. Yalagachin G, Lakshmikantha N and Mashal SB: Prevalence of nodular goiter in patients with breast diseases. J Clin Invest Surg 5: 91-95, 2020.

36. Mehrabani D and Manafi A: Breast implants and breast cancer. World J Plast Surg 1: 62-63, 2012.

37. Rostas JW and Dyess DL: Current operative management of breast cancer: An age of smaller resections and bigger cures. Int J Breast Cancer 2012: 516417, 2012.

38. Franceschini G, Di Leone A, Natale M, Sanchez MA and Masett R: Conservative surgery after neoadjuvant chemotherapy in patients with operable breast cancer. Ann Ital Chir 89: 290, 2018.

39. van Oers $\mathrm{H}$ and Schlebusch L: Indicators of psychological distress and body image disorders in female patients with breast cancer. J Mind Med Sci 7: 179-187, 2020.

40. White J, Achuthan R, Turton P and Lansdown M: Breast conservation surgery: State of the art. Int J Breast Cancer 2011: 107981, 2011.

41. Ursaru M, Jari I, Negru D and Scripcariu V: Local and distant recurrences-a comparative study on conservative and radical surgery for breast cancer. Chirurgia (Bucur) 110: 38-42, 2015.

42. Veronesi U, Cascinelli N, Mariani L, Greco M, Saccozzi R, Luini A, Aguilar M and Marubini E: Twenty-year follow-up of a randomized study comparing breast-conserving surgery with radical mastectomy for early breast cancer. N Engl J Med 347: $1227-1232,2002$ 